\title{
Effect of electrospun polyamide 6 nanofibres on the mechanical properties of a glass fibre/epoxy composite.
}

Bert De Schoenmaker ${ }^{a}$, Sam Van der Heijden ${ }^{a}$, Ives De Baere ${ }^{b}$, Wim Van Paepegem ${ }^{b}$, Karen De Clerck $^{a^{*}}$

${ }^{a}$ Ghent University, Textile Department, Technologiepark 907, 9052 Zwijnaarde, Belgium

${ }^{\mathrm{b}}$ Ghent University, Materials science and engineering , Technologiepark 903, 9052

Zwijnaarde, Belgium

*Corresponding author: +32 926454 11, Karen.DeClerck@ugent.be

Abstract

Recently, several types of nanoparticles are frequently incorporated in reinforced epoxy resin composites. A homogeneous dispersion of these nanoparticles is still a problem. Thermoplastic nanofibrous structures can tackle this dispersion issue. Therefore, this paper investigated the effect of electrospun polyamide 6 nanofibrous structures on the mechanical properties of a glass fibre/epoxy composite. The nanofibres were incorporated in the glass fibre/epoxy composite as stand-alone interlayered structures and directly spun on the glass fibre reinforcement. Both ways of nanofibre incorporation have no negative effect on the impregnation of the epoxy. Moreover, the nanofibres remain well dispersed within the matrix. Incorporation of nanofibres increases the stress at failure in the $0^{\circ}$-direction, the best results are obtained when the nanofibres are directly electrospun on the glass fibres. Optical microscopic images also demonstrate that nanofibres prevent delamination when a $90^{\circ} \mathrm{crack}$ reaches a neighbouring $0^{\circ}$ ply. Furthermore, mode I tests showed a small improvement when a thin nanofibrous structure is deposited directly onto the glass fibres. When the composites are loaded under $45^{\circ}$, it is proven that, for an identical stress, the glass fibre composite with deposited nanofibres has less cracks than when interlayered nanofibrous structures are incorporated. Generally, it can be concluded that the addition of polyamide 6 nanofibres improves some mechanical characteristics of a glass fibre/epoxy composite.

Keywords: Structural composites, Polyamide 6 nanofibres, Matrix cracking, Stress-strain curves, Optical microscopy 


\section{Introduction}

Owing to their light weight and high stiffness and strength, fibre reinforced epoxy resin composites are widely used in industry [1,2]. However, an epoxy matrix is a brittle material, which could lead to unexpected failure of the composite. Thus, an improvement of the resin rich region between two plies with a different fibre orientation is recommended. Therefore, secondary (sub)micron reinforcements are often incorporated in the matrix.

Nanoparticles such as carbon nanotubes (CNT) and nanoclays can be added to the epoxy matrix to improve the mechanical properties of the matrix [3-6]. Due to their theoretical high stiffness and strength, CNT might improve the matrix characteristics [7-9]. However the overall improvement in mechanical properties such as stiffness and fracture toughness of the epoxy matrix is mostly very moderate $[10,11,3]$. The main disadvantage with these CNT's and nanoclays, besides the safety issues due to the small dimensions [12], is the difficulty in obtaining a homogeneous dispersion of the nanoparticles in the resin $[7,13,14]$. Moreover, the viscosity of the resin increases significantly when nanoparticles are added. The toughness of a matrix can also be improved by incorporation of rubber particles or embedding thermoplastic inclusions $[15,16]$. But again, a homogeneous dispersion of the particles in the matrix is hard to obtain.

Thermoplastic nanofibrous structures offer a solution for the dispersion issue, since they can be readily embedded in the resin and incorporate a nanosized phase in the composite. These nanofibres also do not increase the viscosity of the resin. Moreover, due to their macro scale length, no health hazards are involved in the production and use of these nanofibres. Recent literature indicates that nanofibres may contribute substantially to the ductility and fracture toughness of the composites [17-20]. This is related to the hypothesis that a secondary fibrous structure with a pronounced lower fibre diameter in addition to a primary fibre structure may increase several mechanical properties of composite materials [21].

This paper describes the effect of electrospun polyamide 6 (PA 6) nanofibrous structures on the mechanical properties of a glass fibre/epoxy composite. Therefore, the mechanical properties of a pure glass fibre composite are compared to glass fibre composites with added nanofibres. These nanofibrous structures are, on one hand, incorporated between the glass fabrics as stand-alone structures and, on the other hand, directly deposited onto 
the glass fabrics. In addition to the tensile tests, dynamic mechanical analysis and mode I tests using a double cantilever beam are used to examine the properties of the investigated composites. The crack propagation in the loaded sample is studied through optical microscopy.

\section{Materials and methods}

\section{Materials}

All composite plates were reinforced with unidirectional E-glass fabric (Roviglas R17/475). The reinforcement was $475 \mathrm{~g} / \mathrm{m}^{2}$ in the fibre direction, while in the perpendicular direction the reinforcement was $17 \mathrm{~g} / \mathrm{m}^{2}$. The matrix was EPIKOTE resin MGS RIMR 135 with EPIKURE curing agent MGS RIMH 137, both purchased from Hexion (Currently Momentive).

The composite plates were manufactured by vacuum assisted resin transfer moulding (VARTM) using a closed steel mould. The epoxy was first cured at room temperature for 24 hours and, thereafter, post cured for 15 hours at $80^{\circ} \mathrm{C}$, as described by the supplier. For all composite plates the stacking sequence was $\left[0^{\circ}, 90^{\circ}\right]_{2 s}$. The samples were cut to dimensions on a water-cooled diamond saw. All specimens had a thickness of $3.0 \mathrm{~mm}$ and a nominal width of $30 \mathrm{~mm}$, as described in the ISO 527-5:2009. End tabs were used to avoid failure at the clamps.

The nanofibres were incorporated in the glass fibre/epoxy composites in two different ways. On one hand, they were inserted in between the various glass fibre mats as a stand-alone structure. On the other hand, they were directly deposited on one side of the glass fibre reinforcement. Reference composites without nanofibres were also manufactured, to be able to investigate the improvement of the nanofibre addition.

For the fabrication of the nanofibres, 16 wt\% PA 6 was dissolved in a 1:1 solution of $98-100$ v\% formic acid and 98 v\% acetic acid. Both the polymer and solvents were obtained from Sigma-Aldrich and used as received. To obtain large uniform nanofibrous structures, the nanofibres were produced using a multi-nozzle electrospinning set-up. This multi-nozzle method, an in house developed technology [22], diverged from a mono-nozzle set-up only by the number of nozzles, the general methodology itself is identical. Ten nozzles, each fed by a syringe, were placed in two alternating rows in a plate which had movement in the 
transverse direction. In the meantime, a grounded collector was moving in the longitudinal or production direction.

All nanofibrous nonwovens were spun in a conditioned room at $23 \pm 2{ }^{\circ} \mathrm{C}$ and $50 \pm 5 \% \mathrm{RH}$. The tip-to-collector distance was $7 \mathrm{~cm}$ and the flow rate was set at $1.5 \mathrm{~mL} / \mathrm{h}$. The voltage was adapted until a stable process was achieved, this was between 25 and $30 \mathrm{kV}$. The standalone structures were electrospun directly onto an aluminium foil, and afterwards released using water. For the deposited version, the nanofibres were directly electrospun onto the glass fibre mats. The fibre diameter of the interlayered nanofibrous structures was $150 \pm 19$ $\mathrm{nm}$, while for the deposited nanofibrous structures it was $230 \pm 26 \mathrm{~nm}$.

\section{Methods}

Scanning electron microscopy (SEM), a FEI QUANTA 200F system, was used to investigate the cross sections of the composite plates. Prior to the SEM-measurements, the specimens were gold sputter coated (Balzers Union SCD 030). An optical microscope, a Olympus BX51 with a Olympus UC30 camera, was used to visualize cracks on the polished edges of the composites.

The tensile tests on the composites were performed on an electromechanical Instron $5800 \mathrm{R}$ machine with a load cell of $100 \mathrm{kN}$ following the ISO 527-5:2009-standard. The tests were displacement controlled with a speed of $2 \mathrm{~mm} / \mathrm{min}$ and both displacement and load were recorded. All specimens were instrumented with two strain gauges to measure the longitudinal and transversal strain, $\varepsilon_{\mathrm{xx}}$ and $\varepsilon_{\mathrm{yy}}$, respectively. Not all samples were loaded until failure, since some were needed for other experiments.

On the same Instron 5800R, mode I tests were executed following the ISO 25217:2009standard, the double cantilever beam (DCB) test. Two different thicknesses of the deposited nanofibres were used: 5 and $10 \mathrm{~g} / \mathrm{m}^{2}$. The dimensions of the mode I samples were $20 \times 160 \mathrm{x}$ $3 \mathrm{~mm}^{3}$ and the initial delamination length was $50 \mathrm{~mm}$.

The dynamic mechanic analysis (DMA) was executed on a Q800 from TA Instruments. Before the start of the DMA experiments, a complete calibration was carried out, the temperature calibration being performed using an indium standard. Owing to the high modulus of the samples, the experiments were carried out with a single cantilever clamp. The frequency was kept constant at $1 \mathrm{~Hz}$ and the displacement amplitude was set to $20 \mu \mathrm{m}$. The experiments 
started with bringing the DMA-temperature to $30{ }^{\circ} \mathrm{C}$ followed by a equilibration time of 15 min, after which the temperature was raised at $2.5^{\circ} \mathrm{C} / \mathrm{min}$ to $150^{\circ} \mathrm{C}$.

\section{Results and discussion}

\section{Impregnation of the epoxy resin in the nanofibrous structures}

Before starting tensile tests on the composite samples, it needs to be investigated whether the reinforcements are well impregnated, especially the nanofibrous nonwovens. Nanofibrous nonwovens are structures with a high porosity, but also with very small pores, which can hinder the impregnation. Irregularities such as air bubbles could have a negative effect on the mechanical properties of the overall composite. The SEM images in Figure 1 show the good impregnation of the epoxy in both the glass fibres layer and polyamide nanofibres layer. Figure 1B, an unpolished sample broken in liquid nitrogen demonstrates that the individual nanofibres were not damaged during the VARTM-process. Thus, the nanofibre layers have no disadvantages for the impregnation of the epoxy. This good contact between reinforcement and matrix is crucial for the load transfer from the composite to the reinforcement. Figure 1 also demonstrates very good dispersion of the nanofibres within the resin-rich region.

The dimensions of the resin rich region between two glass plies with different fibre orientation depends on the composition of the composite structure. The glass fibre composites without nanofibres had the smallest gap, only $13 \pm 6.4 \mu \mathrm{m}$. Adding nanofibres in between the glass fibre layer causes a pronounced increase of this resin rich region between two glass fibre plies. The gap between two glass fibre layers for the deposited and interlayered secondary reinforced glass fibres composites was $36.4 \pm 6.0 \mu \mathrm{m}$ and $49.3 \pm 7.5$ $\mu \mathrm{m}$, respectively. This increased space between the glass fibre plies means, as a consequence, that the nanofibre addition should be considered as extra separated plies.

\section{Mechanical behaviour}

\section{$\left[0^{\circ}, 90^{\circ}\right]_{2 s}$ stacking sequence}

Figure 2 proves the reproducibility of the specimens used in this research. As shown in the figure, the different stress strain curves with interlayered nanofibres of 7 test specimens, obtained from 2 different composite plates, coincide very well. Hence, it can be concluded 
that there is good reproducibility between different composite plates, which was also the case for the other composite types. Therefore, it was decided to show a representative curve of each composite type (GF: pure glass fibres; NF-I: interlayered nanofibres; NF-D: deposited nanofibres) in the following graphs.

Since it was expected that the addition of nanofibres to the glass fibre composite would improve the mechanical properties, several tensile tests were performed. However, the stress-strain curves in Figure 3 do not directly show an improvement of the stiffness of the composite in the $0^{\circ}$-direction when nanofibres are added to the glass fibre composites. In this $0^{\circ}$-direction the mechanical properties of the glass fibres are dominant compared to those of the thermoplastic nanofibres. Glass fibres have a much higher strength and stiffness compared to the PA 6 nanofibres. Furthermore, the stiffness of thermoplastic nanofibres is around 1 to $2 \mathrm{GPa}$ [23], while the applied epoxy matrix has a stiffness of $3.37 \pm 0.065 \mathrm{GPa}$.

The strain gauge signal was lost at about $2 \%$ longitudinal strain, caused by debonding of the strain gauges, but the load signal was measured until failure. The first column of Table 1 gives the average stresses at failure of the three different composite types. All values are an average of 5 measurements. By adding interlayered nanofibres in between the glass fibre plies, the stress at failure increased from $550 \mathrm{MPa}$ to $581 \mathrm{MPa}$. When the nanofibres are directly deposited onto the glass fibres the stress at failure increases further to $611 \mathrm{MPa}$. Thus, even though the mechanical properties of the nanofibres are inferior compared to the properties of the glass fibres, they improve the failure stress pronouncedly. It seems that the deposited nanofibres even further facilitate the transfer of the load to the primary reinforcement. This can be understood by taking into account that there is a direct contact between the deposited nanofibres and the glass fibres, which improves the load transfer to the glass fibres.

Indeed, Figure 4 clearly shows the difference in crack propagation between the glass fibre composites with and without PA 6 nanofibres. For glass fibre composites without nanofibres, the $90^{\circ}$ crack turns into a delamination when reaching a neighbour $0^{\circ}$ ply, Figure 4.A. Thus, the structure delaminates. When PA 6 nanofibres are added, this delamination effect is much less explicit, Figure 4.B. and C. Hence, it can be concluded that the nanofibres have a toughening effect in the resin rich region between the plies with different fibre orientation. 
This higher fracture toughness is in agreement with the research of Kim and Reneker on PBI nanofibres as reinforcement in an epoxy composite [21].

Table 1 also gives the Young's modulus of the three different composite types; it seems that there is no major increase of the stiffness when nanofibres are added to the glass fibre reinforcement, as already illustrated in Figure 3. Since the stiffness of the composites is especially influenced through the reinforcement with the highest stiffness, it is logical that the stiffness does not increase significantly when PA 6 nanofibres are incorporated as a secondary reinforcement.

The Poisson's ratio of the several composite types also demonstrates that the effect of the incorporated PA 6 nanofibres is negligible, Figure 5. Because of the comparable stiffness of the resin and the PA 6 nanofibres, the nanofibres have no pronounced effect on the dimensional properties of the composite.

The DMA-results in Figure 6 show, however, a significant improvement of the storage modulus in deflection mode when PA 6 nanofibres are incorporated in the region between two plies with a different fibre orientation. This indicates that the flexural stiffness of the composites nevertheless increases by the addition of the nanofibres structures. The highest improvement is obtained with the interlayered nanofibres. The reason of this improvement is probably at least partly due to the small compression of the glass fibre plies. Owing to the addition of PA 6 nanofibres, the region between two plies with different fibre orientation increased. Combined with the identical thickness of the different composite plates, this results in a compression of the glass fibre plies. This compression of the glass fibre plies was the highest for the interlayered nanofibres, since for these the gap between the glass plies with different orientation was the largest. When the temperature is above the glass temperature of the matrix, there is no difference anymore between the different composite types. Above this glass transition temperature, the mechanical properties of the matrix and nanofibres indeed drastically decreases.

Figure 7 shows the force-displacement curves of the composite samples with and without deposited nanofibres. It is clear that the thickest nanofibrous structures decrease the toughness of the composite, while the thinner $5 \mathrm{~g} / \mathrm{m}^{2}$ nanofibrous structures have an advantage. The force needed for the crack propagation is higher for these nanofibre- 
reinforced composites, compared to the pure glass fibre composite. This also indicates that the thickness of the nanofibrous deposition has a pronounced effect on the toughening properties. Table 2 represents the $G_{\mathrm{lc}}$ of the three composite types. This demonstrates clearly that more energy is needed when a thin nanofibrous layer is incorporated, the $G_{I c}$ value increasing by $13.5 \%$. This is 2.75 times more than the reported increases when the nanofibres were incorporated in the composite as a stand-alone structure [24].

\section{$\left[+45^{\circ},-45^{\circ}\right]_{2 s}$ stacking sequence}

To further investigate the effect of nanofibres on the mechanical behaviour of glass fibre composites, tests under $45^{\circ}$ are also performed. By testing the composites under $45^{\circ}$ the mechanical properties of the glass fibres are less dominant compared to the other components. Hence, the advantages of nanofibres should be more pronounced. Figure 8 shows that for the same shear strain, the shear stress of the composites with nanofibre deposited on glass fibres is significantly higher than the glass fibre composites without nanofibres or with interlayered nanofibre mats. Thus, the deposited nanofibres facilitate the load transfer to the glass fibres, resulting in the higher shear stress. Since the interlayered nanofibres have no direct contact with the glass fibres, the load transfer to the fibres is more similar to the plain glass fibre/epoxy composite, hence there is no improvement in shear stress observed.

Figure 9 shows some optical microscopic images of samples with interlayered and deposited PA 6 nanofibres loaded to $40 \mathrm{MPa}$ and then unloaded. $40 \mathrm{MPa}$ was chosen since this was the end of the linear behaviour of the material composited with interlayered nanofibres. From the optical microscopic images, it is clear that the interlayered samples have many more cracks in the glass plies compared to the glass fibre composite with deposited nanofibres. This is in agreement with the curves in Figure 7. It can thus be concluded that the deposited nanofibres transfer the load much better to the primary glass fibre reinforcement.

The shear moduli of the three different composite types are also noted in Table 1 . It is demonstrated that the shear moduli of glass fibre composites with nanofibres are higher compared to the ordinary glass fibre composite.

The higher shear modulus for the with nanofibres deposited glass fibre composite compared to the reference glass fibre composite is also proven with DMA-measurements, which 
measures the flexural modulus, Figure 10 . For each temperature in the range of 0 to $100^{\circ} \mathrm{C}$ the storage modulus of the nanofibre containing composites is higher than the storage modulus of the pure glass fibre composites. Even although the compression of the glass fibre plies will have an influence on the higher stiffness in this flexural modulus, there will also be an effect of the nanofibres. The mechanical properties of the glass fibres are, indeed, less dominant under $45^{\circ}$. This is also in agreement with the shear modulus in Table 1.

\section{Conclusions}

The addition of nanofibres to the glass fibre/epoxy composite has no negative influence on the impregnation of the epoxy in the several types of reinforcement. It was also shown that the dispersion of the nanofibres was good throughout the resin rich region. However, nanofibres affect the dimensions of the resin rich region between the plies with different fibre orientation. The largest gap increase was found for the interlayered nanofibres.

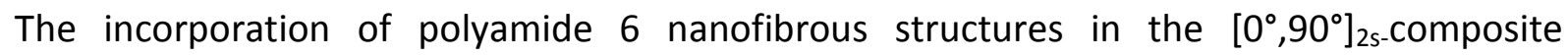
increased the stress at failure, deposited nanofibres were slightly better than the interlayered nanofibrous structures. It was found that nanofibres prevent or minimize the formation of delamination cracks between two glass fibre plies. DMA data showed that the stiffness slightly improves, probably due to a compression of the glass fibre plies.

Mode I tests proved that there is a small improvement of the toughness when nanofibres were incorporated in the glass fibre composite. The thickness of this nanofibre layer was crucial for the properties.

The tensile experiments under $45^{\circ}$ also demonstrated that the deposited nanofibres facilitate the load transfer to the glass fibres. Optical microscopic images showed that at 40 MPa an interlayered nanofibre reinforced glass fibre composite has many more cracks than a deposited one. Thus, it can be concluded that deposited nanofibres improve significantly some mechanical properties of a glass fibre composite. It is suggested that the nanofibre layer form a barrier for crack propagation.

\section{Acknowledgement}

The authors would like to thank David Langendries for his contribution to this work. 


\section{References}

[1] S.T; Peters, Handbook of composites, second edition, Chapman and Hall, 1998

[2] Introduction to composites, reference handbook, The composite institute, 2001, fourth edition

[3] Behnam Ashrafi et al., Enhancement of mechanical performance of epoxy/carbon fiber laminate composites using single walled carbon nanotubes, Composites science and technology, 71(13), 1569-1578, 2011

[4] Betine Nuhiji et al., The effect of alternate heating rates during cure on the structure property relationships of epoxy/MMT clay nanocomposites, Composites science and technology, 71(15), 1761-1768, 2011

[5] Toshio Ogasawara et al., Mechanical properties of aligned multi-walled carbon nanotubes/epoxy composites processed using a hot-melt prepreg method, Composites science and technology, 71(16), 1826-1833, 2011

[6] Konstantinos G. Dassios, Simone Musso, Costas Galiotis, Compressive behaviour of MWCNT/epoxy composite mats, Composites science and technology, 72(9), 1027-1033, 2012

[7] J.N.N Coleman, Small but strong: A review of the mechanical properties of carbon nanotube-polymer composites, Carbon, 44, 1624-1652, 2006

[8] C. Tsu-Wei, An assessment of the science and technology of carbon nanotubre-based fibers and composites, Composite science and technology, 71, 1-19, 2009

[9] H. Qian, E.S; Greenhalgh, M.S.P. Shaffer et al., Carbon nanotube-based hierarchical composites: a review, Journal of Materials chemistry, 20, 4751-4762, 2010

[10] A.T. Seyhan, M. tanoglu, K. Schulte, Mode I and mode II fracture toughness of E-glass non-crimp fabric/ carbon nanotubes (CNT) modified polymer based composites, Engineering fracture mechanics, 75, 5151-5162, 2008

[11] R.J. Sager, P.J. Klein, D.C. Davis, D.C. Davis, G.L. Lagoudas, G.L. Warren, H.J. Sue, Interlaminar fracture toughness of woven fabric composite laminates with carbon nanotube/epoxy interleaf films, Journal of applied polymer science, 121, 2394-2405, 2011 [12] K. Savonlainen et al., Nanotechnologies, engineered nanomaterials and occupational health and safety - a review, Safety science, 48, 957-963, 2010

[13] C. Tsu-Wei, An assessment of the science and technology of carbon nanotubre-based fibers and composites, Composite science and technology, 71, 1-19, 2009

[14] H. Qian, E.S; Greenhalgh, M.S.P. Shaffer et al., Carbon nanotube-based hierarchical composites: a review, Journal of Materials chemistry, 20, 4751-4762, 2010

[15] Pieters R, Miltner HE, Van Assche G, Van Mele B,(2006). Kinetics of temperatureinduced and reaction-induced phase separation studied by modulated temperature DSC. Macromolecular Symposia. 233 (2006), 36-41

[16] McGarry, F. J. In Polymer Toughening, Arends, C. B., ed., Marcel Dekker, Inc.: New York, 1996, pp 175-188

[17] J. Zhang, T. Lin, X. Wang, Electrospun nanofibre toughened carbon/epoxy compoistes: effects of polyetherkertone cardo (PEK-C) nanofibre diameter and interlayer thickness, Composites science and technology, 70, 1660-1666, 2010

[18] A.D. Kelkar, R. Mohan, R. Bolick, S. Shendokar, Effect of nanoparticles and nanofibres on mode I fracture toughness of fiber glass reinforced polymeric matrix composites, Materials science and engineering B, 168, 85-89, 2010

[19] H. Fong, Electrospun nylon 6 nanofiber reinforced bis-gma/tegdma dental restorative composite resins, Polymer, 45, 2427-2432, 2004 
[20] J. Zhang, T. Yang, T. Lin, C.H. Wang, Phase mophology of nanofibres interlayers: critical factor for toughening carbon/epoxy composites, Composites science and technology, 72, 256-262, 2012

[21] J.S. Kim, D.H. Reneker, Mechanical properties of composites using ultrafine electrospun fibres, Polymer composites, 20, 124-131, 1999

[22] P. Westbroek, T. Van Camp, S. De Vrieze, K. De Clerck, PCT/EP2008/056050, 2008

[23] S. Ramakrishna, K. Fujihara, W._E. Teo, T._C. Lim, Z. Ma, An introduction to electrospinning and nanofibres, World scientific publishing co. pte. Ltd., 2005 [24] R. Palazzetti, A; Zucchelli, C. Gualandi, M.L. Focarete, L. Doanati, G; Minak and S. Ramachrishna, Influence of electrospun nylon 6.6 nanofibrous mats on the interlaminar properties of Gr-epoxy composite laminates, Composite structures, 94, 571-579, 2012 